\title{
Prediction of the Coating Thickness of Wire Coating Extrusion Processes Using Artificial Neural Network (ANN)
}

\author{
Bekir Cirak (Corresponding author ) \\ Department of Mechanical Engineering \\ Naturel Science Instutie, Sakarya Unıversity, Adapazarı - TURKEY \\ E-mail: bekircirak@mynet.com.tr \\ Recep Kozan \\ Department of Mechanical Engineering \\ Engineering Faculty, Sakarya Unıversity, Adapazarı - TURKEY \\ E-mail: kazan@sakarya.edu.tr
}

\begin{abstract}
This paper presents a new method of modeling the nonlinear parameters of a coating systems base on neural Networks with artificial neural network neurons. Artificial neural networks (ANNs) are a new type of information processing system based on modeling the neural system of human brain. The wire coating thickness and quality depend on the wire speed, polymer viscosity, polymer melt temperature and the gap between the wire and exit end of the die. In this paper, results of experimental investigation are presented by comparing the coating quality on galvanized mild steel wire using EP 58 PVC molten is used as the coating material in a wire coating extrusion unit at different extruder temperatures and extruder speeds.

The coating thickness and quality are also discussed for different wire speeds of up to $15 \mathrm{~m} / \mathrm{s}$. A three layer back propogation artificial neutral network (ANN) model was used for the description of wire coating thickness.On comparing the experimental data, the predictions the ANN model predictions, it is found that the ANN model is capable of predicting the coating thickness. The neural network model shows how the significant parameters influencing thickness can be found. Inthis studies, a back propagation neural network model is developed to map the complex non-linear wire coating thickness between process conditions .
\end{abstract}

Keywords: Wire coating extruder, Coating thickness, Artificial neural network (ANN), PVC, Extrusion

\section{Introduction}

Polymer coating is often applied to wires, strips, tubes or ropes for insulation or protection against corrosion. There are three different methods which are mostly used for this coating process. These methods are coaxial extrusion, dipping and electro-statical deposition process. The first two processes can be reasonably fast but bonding between the continuum and the coating material is not so strong. The third process offers much stronger bonding but is relatively slow. If the coating material can be forced onto the continuum uniformly the bonding can be improved significantly.

Artificial neural network (ANN) computing was originally inspired by the operations of biological brains. It was developed to simulate the strong learning, clustering and reasoning capacity of biological neurons.With a strong learning capability and use of parallel computation and nonlinear mapping, neural networks can be successfully applied to identifying several nonlinear systems and control problems.

The analysis for different coating processes could be found in the literature. Hydrodynamic wire coating with Nylon 6 using a tapered bore pressure unit has been presented.(B. Caswell, R.I. Tanner, 1978)(C.L. Tucker, 1989)(H. Zhang, M.K. Moallemi, S. Kumar, 1991)

Coextrusion method for coating has been studied theoretically and experimentally. (S. Nakazawa, J.F.T. Pittman, 1982)(V.S. Mirnov, 1993)

Present study of dip coating process. An experimental study of the electro-statical deposition process has been presented.

A continuously increasing number of commercial products are produced by polymer extrusion using plasticating extruders, which are among the most widely used equipments in polymer process industry. The extrusion process has a 
standard setup includinga feeding section, a barrel and a head with a die for shaping. In the feeding section, the solid polymer is fed into the extruder through a hopper in the form of pellets or irregular small bits. Then, the polymer is transported along the barrel by means of a rotating screw.

A continuously increasing number of commercial products are produced by polymer extrusion using plasticating extruders, which are among the most widely used equipments in polymer process industry.

The extrusion process has a standard setup including a feeding section, a barrel and a head with a die for shaping. In the feeding section, the solid polymer is fed into the extruder through a hopper in the form of pelletsor irregular small bits. Then, the polymer is transported along the barrel by means of a rotating screw. The barrel wall is equipped with a number of electric heaters which melt the polymer. The material is melted and pushed towards the die where the extruded final product is shaped and expelled. During the process, the polymer undergoes very complex thermo-mechanical transformations inducing strong changes in the physical properties of the material.(Ming-Der Jean, Chyuan-Du Liu, Jen-Ting Wang, 2005)

A high-quality extrusion is essentially characterized by a precisely-regulated output volumetric flow; this can be achieved by finely regulating the temperature and the pressure of the die at the output of the extruder (Noriega, del pilar, \& Rauwendaal, 2001).(Hulya Kacar Durmus, Erdogan Ozkaya,Cevdet Meric, 2004)

Paste extrusion is widely used in the food, chemical, ceramic and pharmaceutical industries. Recently, issues arise in the electrical industry for manufacturing superconducting wire and for carrying out electrical insulation of superconducting cables by extrusion. It can thereby be seen that the role of paste extrusion is important in many industries. The process generally involves paste preparation, die extrusion, drying, and firing in order to create a final product. Benbow and Bridgwater have summarised the stages for forming products. Paste flow and the material properties are crucial factors for product quality. Studies including the effect of the particle size binder material, carbohydrate, liquid-phase rheology and mixing procedure on the extrusion process and the extrudate have been carried out.(C.D. Han, D. Rao, 1978)

The barrel wall is equipped with a number of electric heaters which melt the polymer. The material is melted and pushed towards the die where the extruded final product is shaped and expelled. During the process, the polymer coating is often applied to wires, strips, tubes or ropes for insulation or protection against corrosion. (fig.3 )

The plasticating extruder is one of the main pieces of equipment used in the polymer processing industries. As plastics is found more uses, with more stringent quality specifications, the methods of increasing polymer production while improving product quality are needed. Extrusion molding is the most widely used process in manufacturing plastic products. Since the quality of extrusion coated plastic parts are mostly influenced by process conditions, how to determine the optimum process conditions becomes the key to improving the part quality.(S. Basu, 1981)

\section{Material and experimental procedure}

In this study, EP 58 PVC coating plastic material was used as test material. The material was supplied from EL-KI KABLO (Manisa/Turkey). The experimental value and datas is given from this company ( Table 1 )

The die heating zones were maintained at the same temperature, which was also varied during the experiments. The some of temperatures for each heating zone (barrel) and the different screw speeds are shown in Table 1. Sixty combinations were tested for their effect on the parameters of the extruded parts. The process settings were varied randomly and continuously; i.e. without stopping the process. Each setting was allowed to run. Table 1 shows first 30 experimental value used in training and other 30 experimental value used in testing.

To measure the coating thickness, each sample is measured at five different positions along its length by a micrometer and the average diameter of the coated wire is noted. A small portion of the wire sample is cold mounted and polished. An optical microscope is then used to assess the actual coating thickness and the concentricity of the coating on the wire. Fig.1. shows geometry model and this model to consist of an optical microscope.

The geometry gives distance of coating centre between wire centre of wire coating section.In this paper, the wire coating states of an industry example, shown in Fig. 2, was studied. The polymer material used for coating the cover is PVC.

Also the geometry gives distance of coating centre between wire centre of wire coating section. The other words, the eccentricity (unüniform coating ) and concentricity (üniform coating) of the wire coating states of an industry example, shown in Fig.3 was studied. Fig.3 shows the state of eccentricity of the coating on wire with wire velocity of $2050 \mathrm{~d} / \mathrm{dk}$ $(15 \mathrm{~m} / \mathrm{s})$ and extruder temperature of $150{ }^{\circ} \mathrm{C}$. Fig. - b shows the quality of concentricity of coating at wire velocity of $2065 \mathrm{~d} / \mathrm{dk}(19 \mathrm{~m} / \mathrm{s})$ and extruder temperature of $175{ }^{\circ}$ C.The coating is not absolutely concentric but for general application is considered to be satisfactory.

The experimental set up consists of the drawing bench, the wire bobbine, the cooling pool, the drive system (electrical motor), the polymer feeding and melting unit (extruder) and the drawer and winder unit. A schematic diagram of the process is shown in Fig. 4. 
The polymer granules are filled in the hopper and the hopper is connected to the body of extruder.

The process parameters for the investigations carried out were as follows. Polymer characteristics Polymer type EP 58 PVC, Polymer melt temperature $150-200{ }^{\circ} \mathrm{C}$ and Wire characteristics Wire diameter $1,40 \mathrm{~mm}(0,20 \times 7 \mathrm{~mm})$, Wire material Galvanized mild steel. Experimental work was carried out at polymer melt temperatures of 150, 185 and 200 ${ }^{\circ} \mathrm{C}$.

A pvc compound was prepared using a proprietary formula which includes PVC resin, foaming (blowing) agent, heat stabilizer, lubricant, process aid, and filler. The extrusion process parameters; i.e. barrel heating zones' temperatures and screw speed were varied systematically and in random order to vary of the extruded parts.(S. Akter, M. S. J. Hashmi, 2006)

The available extruder incorporated three heating zones along the barrel and two independent heating zones at the die sections. In order to maintain a constant heat profile in the barrel, the ratio between the three heating zones was fixed during all experiments while varying the temperatures of all three barrel zones accordingly.

\section{Artificial neural networks}

In recent years artificial neural networks (ANNs) have emerged as a new branch of computing, suitable for applications in a wide range of fields. Artificial neural networks have been recently introduced into plastic extrusion.(Geng-Qun Huang, Han-Xiong Huang, 2007)

In this study, experimental and ANNs results have been compared. A lot of studies have been published in which the prediction of various parameters on coating thickness were investigated systematically.S.Akter,M.S.J. Hashimi of paper is presents a new neural network approach to polymer coating extrusion.(B. Caswell, R.I. Tanner, 1978)

Among the several proposed types of artificial neural network models, the back propagation network is the most extensively used learning algorithm that includes nodes with continuously differentiable activation functions. Each node performs a particular nonlinear operation that involves a weighted sum of its inputs. Its outputs are fed into trained nodes. The error at each neuron or node is calculated and the weight for each neuron is modified until the desired error between the actual and the required output is achieved. A well-trained ANN can be used to simulate and predict the output response for various control factor-level settings. (Geng-Qun Huang, Han-Xiong Huang, 2007)

ANN method is proposed to optimize the polimer extrusion process. In this method, a back propogation neural network model is developed to map the complex non-linear relationship between process conditions and quality indexesof the extrusion coated parts.

In this study, we use a three-layered back propagation network that includes an input layer, a hidden layer and an output layer shown in Fig.5, Fig.6 and Fig.7.

Artificial neural network (ANN) is a data modeling tool that is able to capture and represent the complex relationship of the input and output data. Back-propagation neural network is the most widely used neural network. It has feed forward network architecture constituted by a set of processing units, also known as neurons, which are arranged in a layered structure. The connections between the units are weights, which are used to store the knowledge of the network. The Back-propagation neural network has been applied in many engineering applications.(Nidal H. Abu-Zahra, Ashish Seth, 2002)

A back propagation can make accurate predictions when applied to the coating treatment process because it can model complex nonlinear, multiple functional relationships using training and testing samples for self-learning and recalling.

\subsection{Structure of an ANN model for coating thickness}

Many different process parameters affect the quality of the extrusion molded products. Variables with greater influence on the part quality need to be selected in order to simplify the problem by saving both the sample collecting time and the computing time.

In this paper, the selection of process variables as inputs of ANN model is based on the relative significance of each variable on the objective performance. The neuron number of the input layer of ANN is determined by the number of variables selected, and the neuron number of the output layer is determined by the number of the objective indexes.

In this paper, a three-layer ANN model with one hidden layer was used, where the neuron number of the hidden layer was determined by trials. The transfer function between the input layer and the hidden layer is 'Tansig', while the transfer function between the hidden layer and the output layer is 'Purelin'.

The architecture of a neural network depends on its network topology, transfer function, and learning algorithm. A typical Back-propagation neural network, as shown in Fig. 4, is composed of three layers, namely, input layer, hidden layer, and output layer. (Shen Changyu, Wang Lixia, Li Qian, 2007) 
ANNs are widely accepted as a technology offering an alternative way to simulate complex and ill-defined problems. They have been used in diverse applications in control, robotics, pattern recognition, forecasting, power systems, manufacturing, optimization, signal processing, etc., and they are particularly useful in system modeling.

A neural network is a computational structure, consisting of a number of highly interconnected processing units called neurons. The neurons sum weighted inputs and then applies a linear or non-linear unction to the resulting sum to determine the output and the neurons are arranged in layers and are combined through excessive connectivity. (Nidal H. Abu-Zahra, Ashish Seth, 2002)

Back propagation network is a typical ANN that has been widely used in many research fields. Back propagation network have hierarchical feed forward network architecture, and the outputs of each layer are sent directly to each neuron in the layer above.(Ming-Der Jean, Chyuan-Du Liu, Jen-Ting Wang, 2005)

Back propagation network are trained by repeatedly presenting a series of input/output pattern sets to the network. The neural network gradually 'learns' the governing relationship in the data set by adjusting the weights between its neurons to minimize the error between the actual and predicted output patterns of the training set. A separate set of data called the test set is usually used to monitor network's performance.

When the mean squared error (MSE) of the test set reaches a minimum, network training is considered complete and the weights are fixed. In essence, a neural network is a function that maps input vectors to output vectors.(Nidal $\mathrm{H}$. Abu-Zahra, Ashish Seth, 2002)

The outputs of the neurons in the input layer are same as their inputs shown in Eq. (1) for the hidden layer, each neuron receives total outputs from all of the neurons in the input layer as

$$
\text { net }_{j}=\sum_{i=1}^{n} w_{i j} x_{i}+\theta_{j}
$$

where net $j$ is the net input to the $j$ th neuron in the hidden layer, $n$ the number of neurons in the input layer, wij the connection weight from the $i$ th neuron in the input layer to the $j$ th neuron in the hidden layer, $x i$ the input to the $i$ th neuron in the input layer, and $\theta j$ is the threshold value of the $j$ th neuron in the hidden layer.

The output of a neuron in the hidden layer is calculated by applying the net input to a transfer function. The sigmoid function shown in Eq. (2) was used as the transfer functions between input layer and hidden layer.

$$
y_{j}=f\left(\text { net }_{j}\right)=\frac{1}{1+\mathrm{e}^{\text {net }_{j}}}
$$

For the output layer, the output of the neurons is shown in Eq. (3)

$$
o_{k}=\sum_{j=1}^{m} w_{j k} y_{j}+\theta_{k}
$$

where $o k$ is the output of the $k$ th neuron in the output layer, $m$ the number of neurons in the hidden layer, wjk the connection weight from the $i$ th neuron in the hidden layer to the $k$ th neuron in the output layer, $y j$ the output from the $j$ th neuron in the hidden layer, and $\theta k$ is the threshold value of the $k$ th neuron in the output layer.(Shen Changyu, Wang Lixia, Li Qian, 2007)

In this work, a back propagation neural network model was developed to build the relationship between parison thickness distribution and the objective function. The thicknesses of 0.5 positions arranging uniformly along the parison were selected as input parameters for the ANN model. The output parameter was the objective function value.

The architecture of a neural network depends on its network topology, transfer function, and learning algorithm. A typical back propagation neural network, as shown in Fig. 8, is composed of three layers, namely, input layer, hidden layer, and output layer.

There is no definite rule available to determine the appropriate number of neurons in the hidden layer. In this paper, it was determined by a trial and error method. The results showed that the ANN model with 13 neurons in the hidden layer could give a better converging rate and generalization. The architecture of the ANN model used in this work is shown in Fig.8. 
The ANN model for the coating thickness variation of the part built by the above methods is shown in Fig. 8 . The node number of the hidden layer was determined by train trials and the final value obtained was 9, that made the configuration of ANN as 6-9-1.

A neural network system is presented for use in wire coating process. The tan-sigmoid transfer function was used as the activation function for the hidden layers, and linear transfer function was used for the output layers. Network architecture for coating thickness and the contrast of prediction results and numerical experiment results of relationship between coating thickness variation and process parameters are shown in Fig. 8.

All the input and target data were scaled in the range between [0.1,0.9] using the following normalization equation shown in Eq. (4)

$$
V^{\prime}=\frac{V-V_{\min }}{V_{\max }-V_{\min }} \times 0.8+0.1
$$

where $V$ is the original data, $V \min$ and $V \max$ the minimum and maximum values of $V$, respectively, and $V$ is the normalized data of the corresponding $V$. Totally 300 patterns were obtained from numerical simulation by changing the thickness distribution of the coating surface along its length.

Among them, 270 patterns were used as training ones. Once the mean square error (MSE) for the training patterns reduced within a given tolerance (set at 10-6 in this work), or the number of training iterations reached a predetermined one (set at 1000 in thiswork), the network-training course was stopped. Fig.9 shows iteration number versus mean square error for training nonlinear coating thickness.

The results of training and testing are confirmed experimentally. According to the back propagation models, the R2 for training and for testing is 0.924 and 0.822 , respectively, which means that the experimental hardfacing roughness values and their predicted values are strongly linear for both models. The values predicted by the back propagation model analysis yield a best-fit average percentage error of $4.61 \%$ from the actual data.

Figs. 10 show the development of training and testing RMS errors for 8-18-1 and 8-18-18-1 neural models over 10,000 learning epochs

The 8-18-1 neural network models, which exhibit well-trained network performance, are superior to the other networks used because the RMS errors for training and testing are rapidly minimized, converging to 0.035 and 0.061 following after 4400 learning epochs, respectively.

Fig. 11 shows the evolution of the mean square error of the ANN model during training. The trained network model was validated for its predictive capability.The result indicates that the ANN has a good performance graphics in Fig. 11.

\subsection{Train/test samples}

This paper deals with the ability of the varied step of each variable is set to obtain a series of set-point values of variables inside the varied range based on baseline. The samples of all set-points are then assigned as input-output data to train and test the ANN. The samples are randomly divided into two groups.

Those samples including the baseline set were methodically assigned to the training set, and all the remaining samples were assigned to the test set. After being trained, the ANN model can map the non-linear relationship between quality indexes and variables of injection molding. It can then be used in the optimization of online process conditions and the part quality control of molded products.

The contrast of prediction results and numerical experiment results of relationship between coating thickness variation and process parameters are show in Fig. 10. The result indicates that the ANN has a good performance, and it can accurately map the relationship between the volumetric shrinkage variation and the process parameters. First 30 experimental value used in Training and other 30 experimental value used in Testing at table 1.

The simulation function based on the ANN was used as the objective function of the optimization problem, and the process window for each variable as given above was used as the boundary restrictions.

The remaining 89 samples were then used to test and to train the performance of the ANN. As shown in Fig. 12,13 most of the samples have consistent outputs of ANN prediction and numerical experiment, and the result is quite satisfactory. Once the optimal ANNs was designed, the ANNs were trained and used to predict coating thickness for various set of environmental conditions (Figs.14).

The results obtained in ANNs application are close to test results. Therefore by using trained ANNs values, the intermediate results that were not obtained in the tests can be calculated. Two value have been selected for test phase (Figs.15). 
The number of the hidden layer with 18 neurons is obtained from Table 2. Table 2 reveals that the suitable number of neurons in the hidden layer is determined by trial and error. However, 11 neural network constructs are obtained using the MATLAB1 interface. The 6-9-1 and 6-13-1 neural models both have small and stable RMS errors for various constructs, respectively.

Fig. 16. shows the experimental data and the predictions of the coating thickness by employing an ANN The process of extrusion was carried out with a sequence of eight extruder velocities, 505, 500, 495, 490, 485, 480, 475 and $470 \mathrm{rpm}$ represented by $\mathrm{v} 1$ to $\mathrm{v} 8$, respectively.

Fig. 17 and 18. Shows experimental data and predictions of extruder screw speed with coating thickness by the $6-13-$ 1 ANN model. Also experimental data and predictions of extruder screw speed with coating thickness by the $6-9-1$ ANN model. 6-13-1 ANN model is prefer one of this models.

Fig. 19 shows the drawing force at a temperature of $150^{\circ} \mathrm{C}$. The theoretical drawing force has also been shown in this figure. The drawing force generally increases with the increase in the wire velocity. The theoretical drawing force matches well with the experimental force though it is slightly higher in magnitude.

Fig. 20 shows the coating thickness for ep 58 pvc at the extruder temperature of $150{ }^{\circ} \mathrm{C}$. These tests were carried out at wire velocities of up to $16 \mathrm{~m} / \mathrm{s}$. The coating was continuous and the thickness was uniform at $0.05 \mathrm{~mm}$ over the entire range of velocities. The pvc melt started to burn as the melt temperature increased beyond $120{ }^{\circ} \mathrm{C}$. This degradation of polymer led to slightly lower quality coating at higher wire velocities.

However, the coating on the polymer for different ranges of velocities, and melt temperatures appeared to be concentric. Some samples were prepared and polished for examination under an optical microscope. The cross section of the wire was observed to be circular and the coat ing was reasonably concentric.

Fig.3-a illustrates the coating concentricity at extruder temperature $175{ }^{\circ} \mathrm{C}$ and wire velocity of $19 \mathrm{~m} / \mathrm{s}$ which appears to be slightly eccentric. Fig.3-b shows the coating is reasonably concentric. Results the quality of concentricity of the coating on wire with extruder temperature $150{ }^{\circ} \mathrm{C}$ and wire velocity of $15 \mathrm{~m} / \mathrm{s}$.

\section{Conclusion}

This paper has presented knowledge based and neural network approaches to wire coating for polymer extrusion. Experimental results of wire coating extrusion with EP 58 PVC have been presented. Due to some limitation in the present experimental set up the drawer speed is limited to about $16 \mathrm{~m} / \mathrm{s}$. Therefore, experiments were carried out within the speed range of up to $15 \mathrm{~m} / \mathrm{s}$. The polymer coating on the wire is continuous for speeds of up to $15 \mathrm{~m} / \mathrm{s}$.

The bonding quality of the coating with wire was found to be very good. Concentricity is beter in the case of extruder with screw speed $485 \mathrm{rpm}$ and wire velocity $2050 \mathrm{rpm}(15 \mathrm{~m} / \mathrm{s}$.) Application of screw speed and wire velocity (drawer speed ) generally improves the quality of the coating.

Conventional wire coating with EP 58 PVC using a tapered bore die unit has been presented. The experiments were carried out within the speed range between 2 and $15 \mathrm{~m} / \mathrm{s}$. The polymer coating on the wire is continuous and concentric for speeds of up to $16 \mathrm{~m} / \mathrm{s}$. The concentricity quality of the coating with wire was very good.

\section{References}

B. Caswell, R.I. Tanner, (1978). Wire coating die design using finite element methods, Polym. Eng. Sci. 18 (5(April)) 417-421.

C.D. Han, D. Rao, (1978). Studies on wire coating extrusion. 1. The rheology of wire coating extrusion, J. Polym. Eng. Sci. 18 (October) 1019-1029.

C.L. Tucker, (1989). Computer Modelling for Polymer Processing, Hanser Publishers, Munich, Vienna, New York, pp. 311-317.

Geng-Qun Huang, Han-Xiong Huang, (2007). Optimizing parison thickness for extrusion blow molding by hybrid method, Journal of Materials Processing Technology. pp. 512-518 Center for Polymer Processing Equipment, China

H. Zhang, M.K. Moallemi, S. Kumar, (1991). Thermal analysis of the hot dipcoating process, journal of heat transfer in metals and container less processing and manufacturing, ASME 49-55.

Hulya Kacar Durmus, Erdogan Ozkaya,Cevdet Meric, (2004). The use of neural networks for the prediction of wear loss and surface roughness of AA 6351 aluminium alloy, department of mechanical engineering. p.p. 201-205 Celal Bayar University, Manisa / Turkey

Ming-Der Jean, Chyuan-Du Liu, Jen-Ting Wang, (2005). Design and development of artificial neural networks fordepositing powders in coating treatment, applied surface science. p.p. 290-303 Computer Science, and Statistics, State University of New York USA 
Nidal H. Abu-Zahra, Ashish Seth, (2002). In-process density control of extruded foam PVC using wavelet packet analysis of ultrasound waves, Industrial and Manufacturing Engineering Department, University of Wisconsin-Milwaukee, pp. 1083-1095 USA

S. Akter, M . S . J . Hashmi, ( 2006 ). wire drawing and coating using a combined geometry hydrodynamic unit : theory and experiment, journal of materials processing technology, pp. 98 - 110 Ireland

S. Basu, (1981). A theoretical analysis of non-isothermal flow in wire-coating co-extrusion dies, J. Polym. Eng. Sci. 21 (December) 1128-1137.

S. Nakazawa, J.F.T. Pittman, (1982). A finite element system for analysis of melt flow and heat transfer in polymer process, in: Numerical Methods in Industrial Forming Process, Pineridge Press, Swansea, UK, 1982, pp. 523-533.

Shen Changyu, Wang Lixia, Li Qian. (2007). Optimization of injection molding process parameters using combinationof artificial neural network and genetic algorithm method, National Engineering and Research Center for Advanced Polymer Processing Technology. (2007). pp. 412-418 Zhengzhou University, China

V.S. Mirnov. (1993). Increase of polymer coating wear resistance by electrophysical modification, in: P.K. Datta, J.S. Gray (Eds.), Surface Engineering: Engineering Applications, vol. II, The Royal Society of Chemistry, 1993, pp. 80-90.

Table 1. Range of experimental process parameters

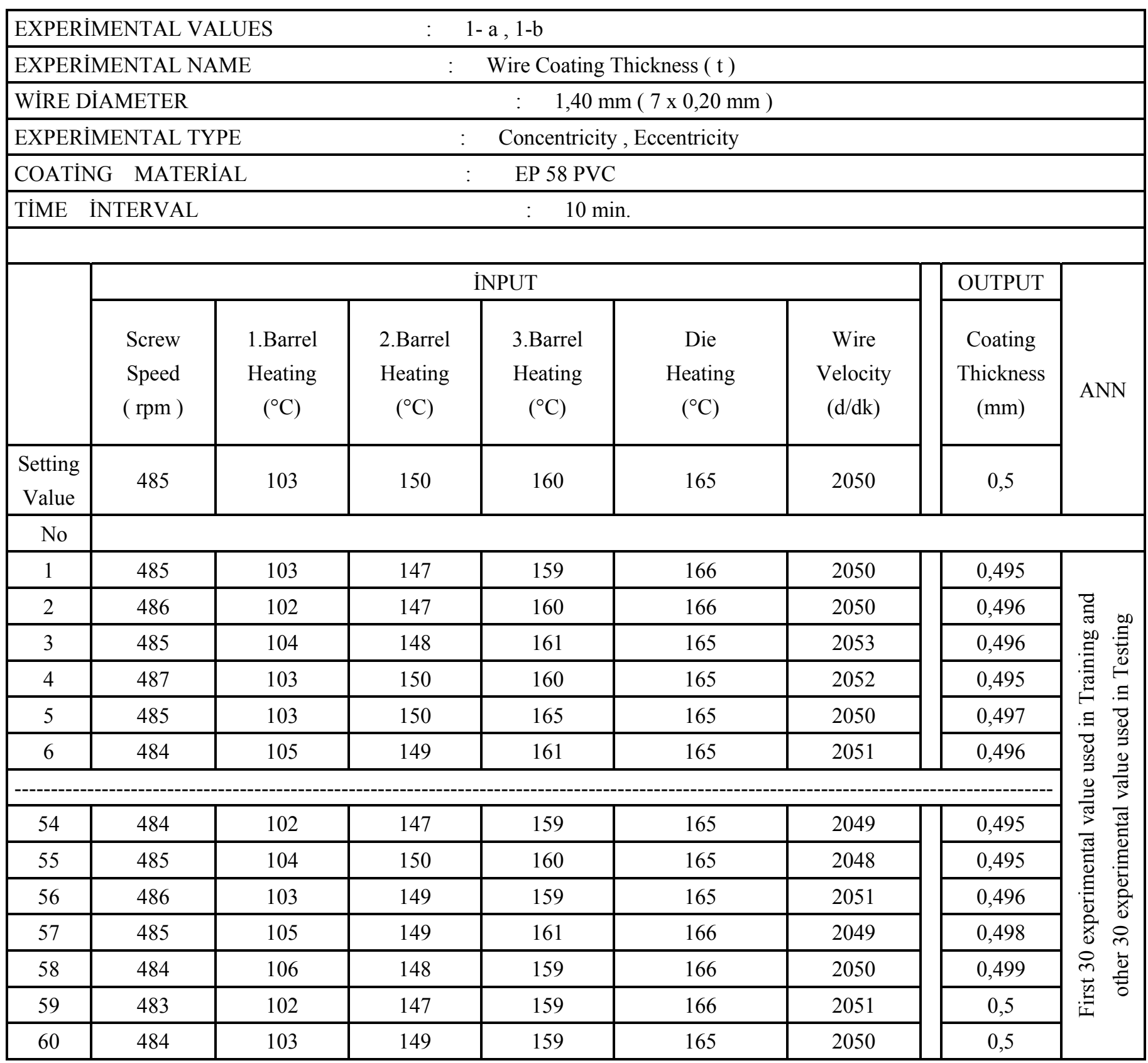


Table 2. The various RMS errors of ANN models through learning epochs

\begin{tabular}{cccc}
\hline Number of trials & Neuron of input-hidden-output & RMS error of training sample & RMS error of testing sample \\
\hline 1 & $6-8-1$ & 0.03482 & 0.06143 \\
2 & $6-9-1$ & 0.03482 & 0.06168 \\
3 & $6-10-1$ & 0.03482 & 0.06210 \\
4 & $6-11-1$ & 0.03482 & 0.06151 \\
5 & $6-12-1$ & 0.03481 & 0.06164 \\
6 & $6-13-1$ & 0.03481 & 0.06158 \\
7 & $6-14-1$ & 0.03481 & 0.06165 \\
8 & $6-15-1$ & 0.03485 & 0.06157 \\
9 & $6-16-1$ & 0.03483 & 0.06166 \\
10 & $6-17-1$ & 0.03482 & 0.06157 \\
11 & $6-18-1$ & 0.03483 & 0.06161 \\
\hline
\end{tabular}
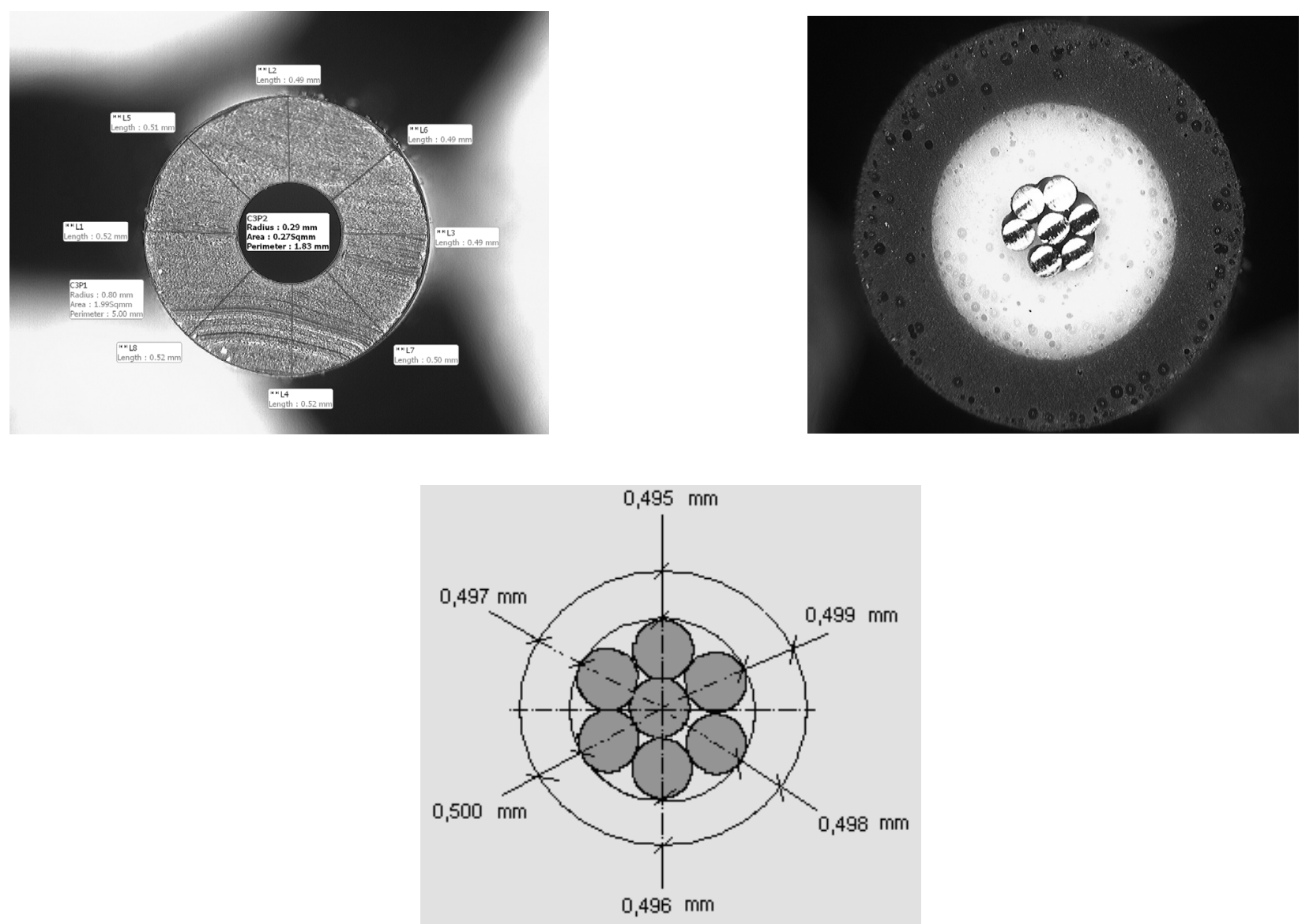

Figure 1. Geometry model 

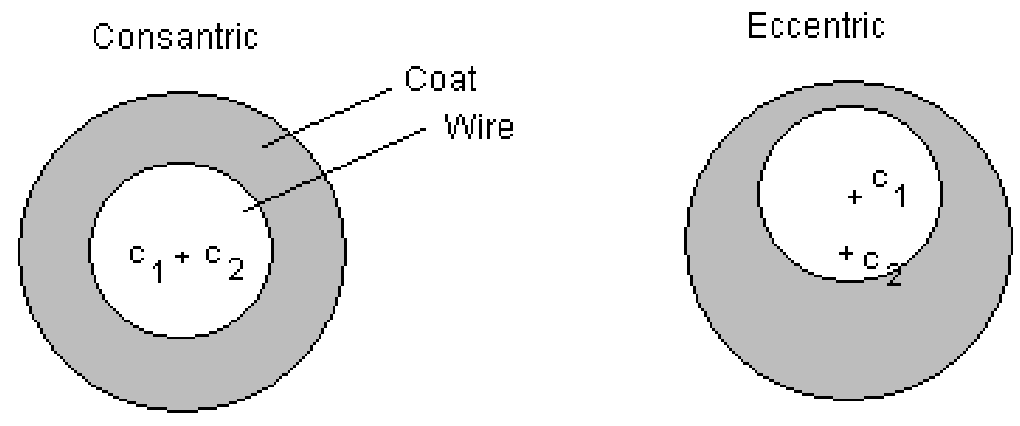

Figure 2. The relationship between centres of wire and coating
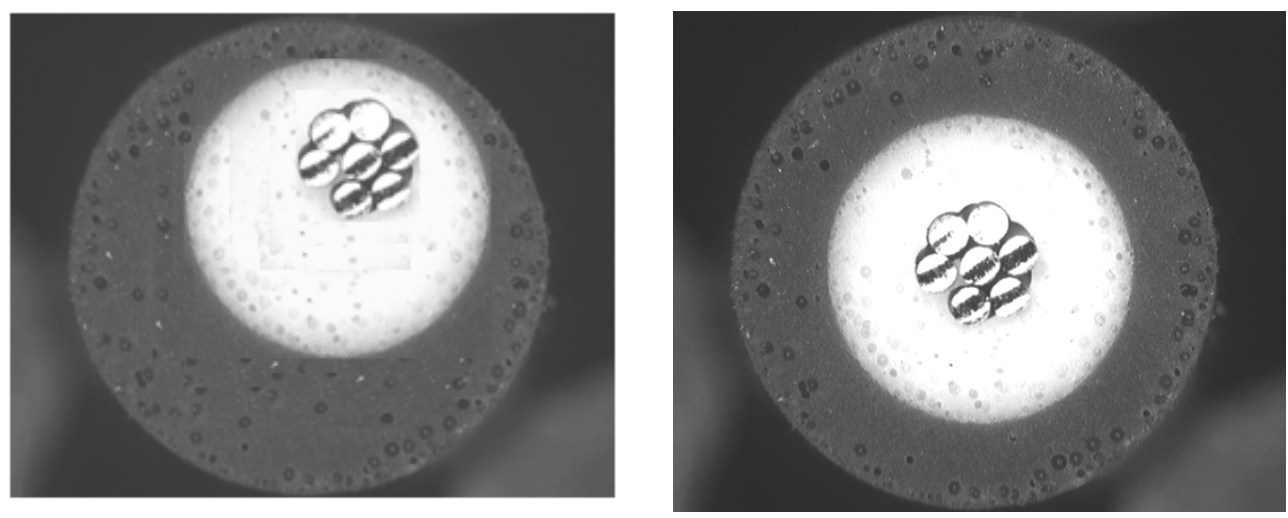

a- Eccentricity state

b- Concentricity state

Figure 3. Wire coating states

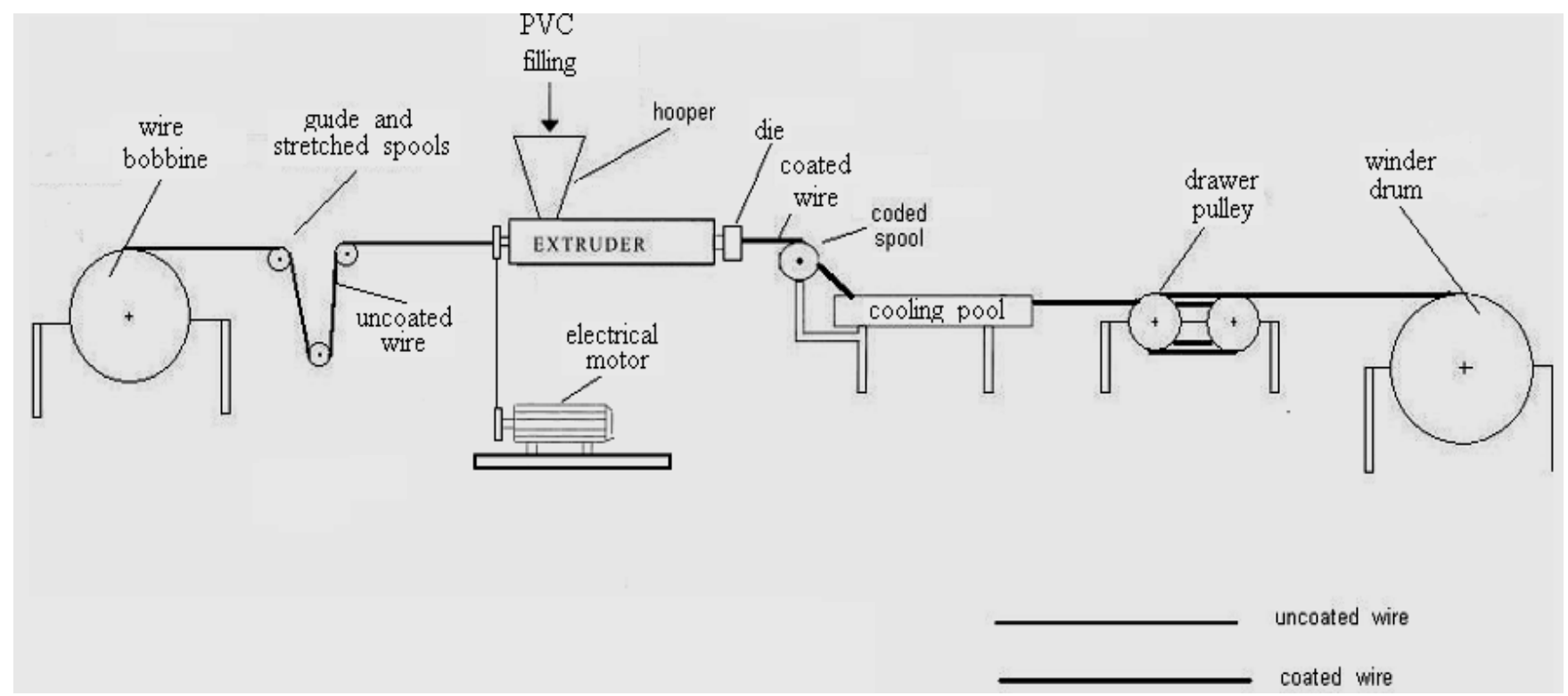

Figure 4. Wire coating extrusion process scheme 


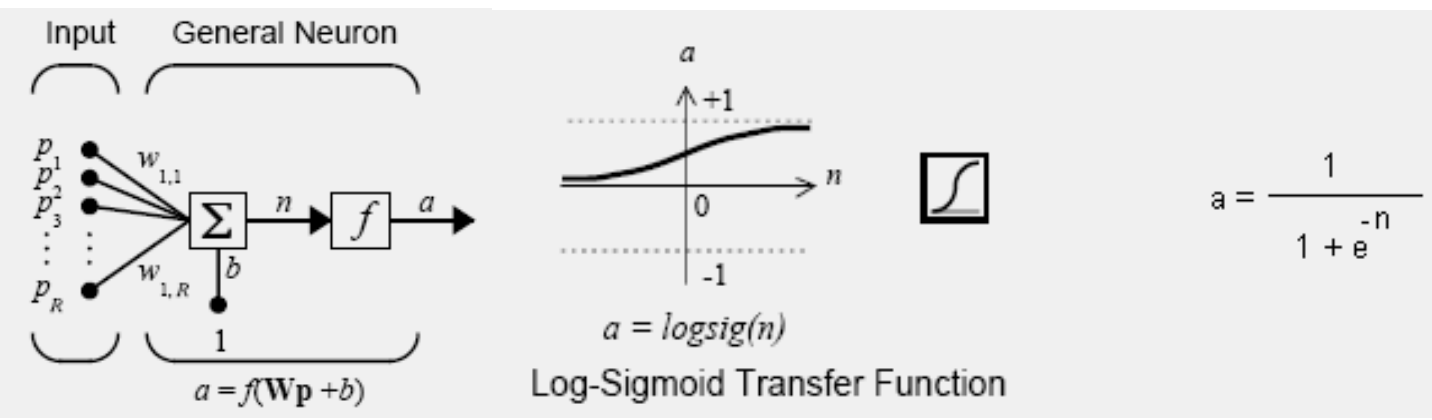

Figure 5. input schematic construction of artificial neural network

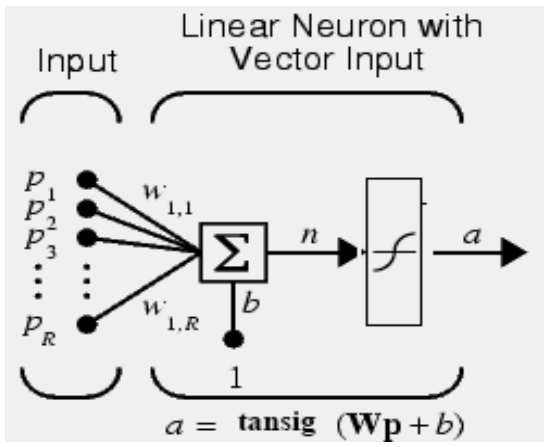

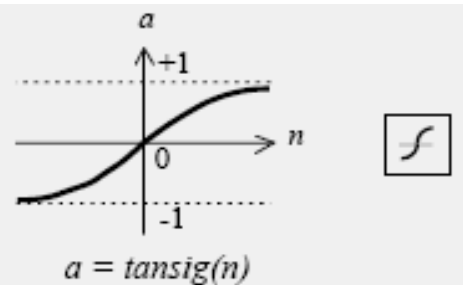

Tan-Sigmoid Transfer Function

Figure 6. hidden schematic construction of artificial neural network

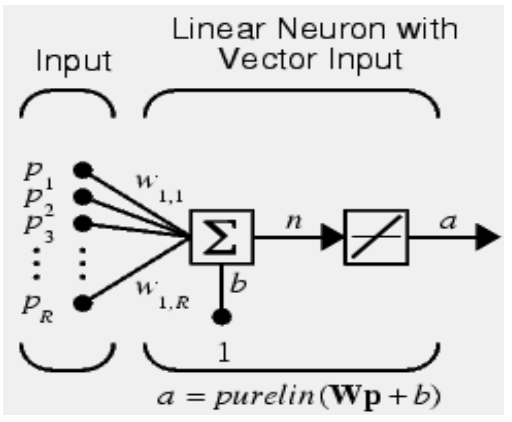

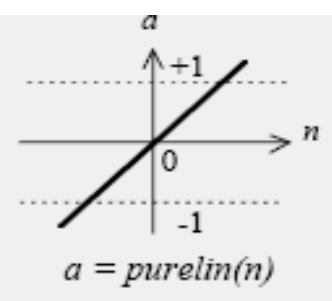

Linear Transfer Function

Figure 7. output schematic construction of artificial neural network 


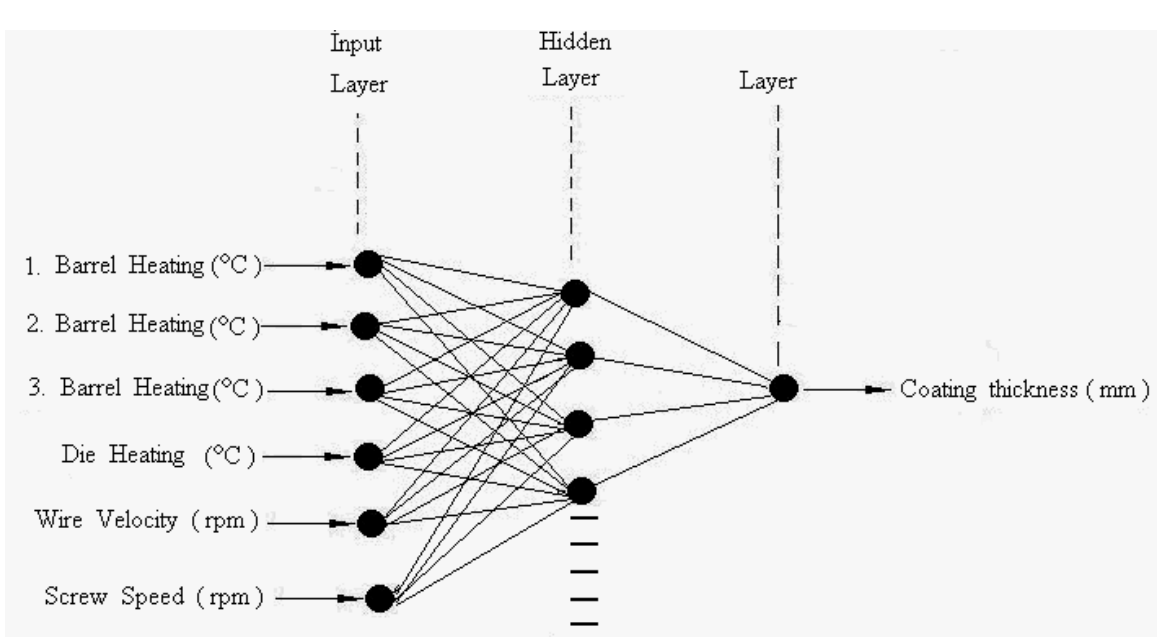

Figure 8. Wire coating configüration of theANN Model

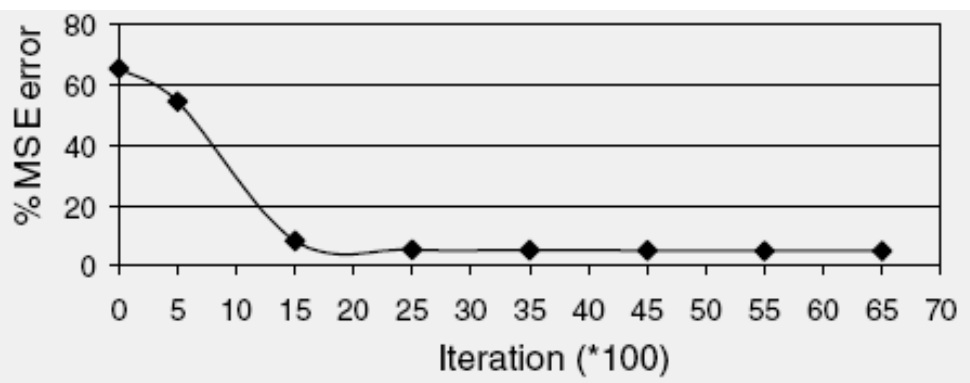

Figure 9. Iteration number versus mean square error for training nonlinear coating thickness

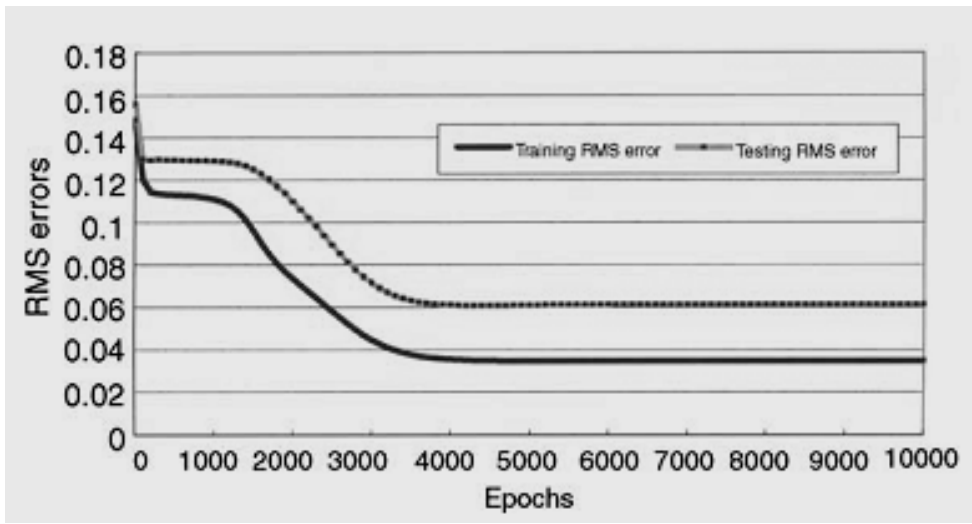

Figure 10. The development of training and testing RMS with learning epochs fort he 6-13-1 ANN model 


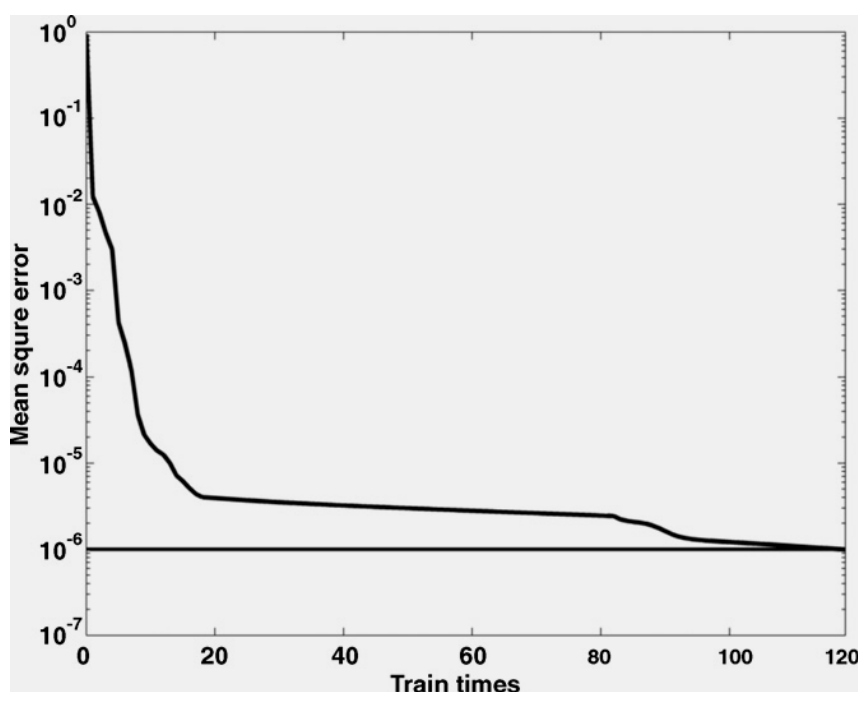

Figure 11. Train eror during the training

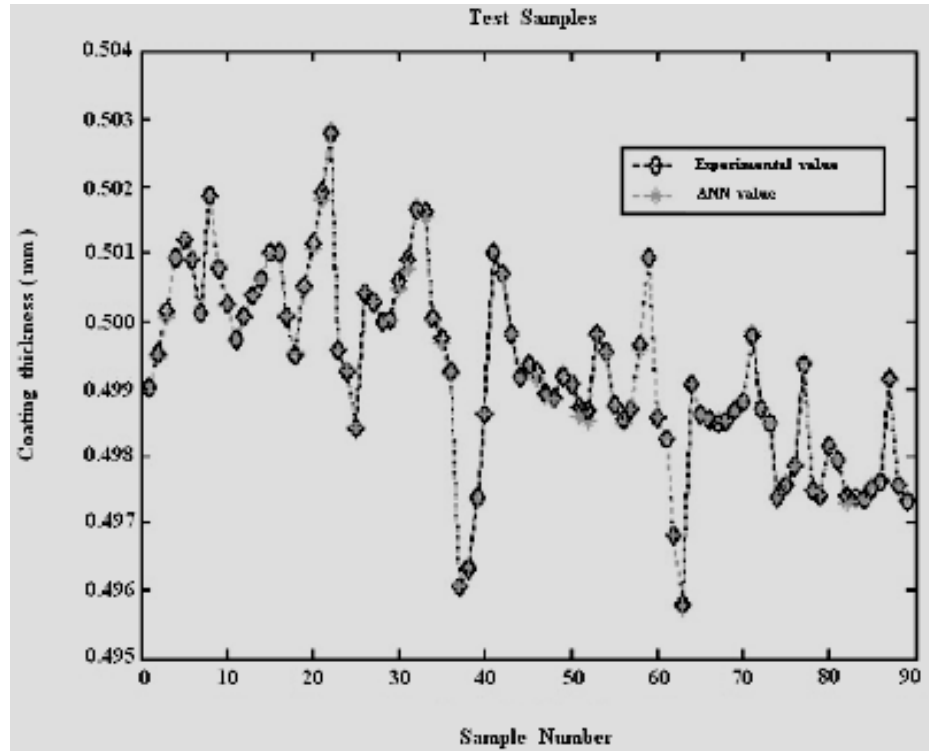

Figure 12. Test samples 


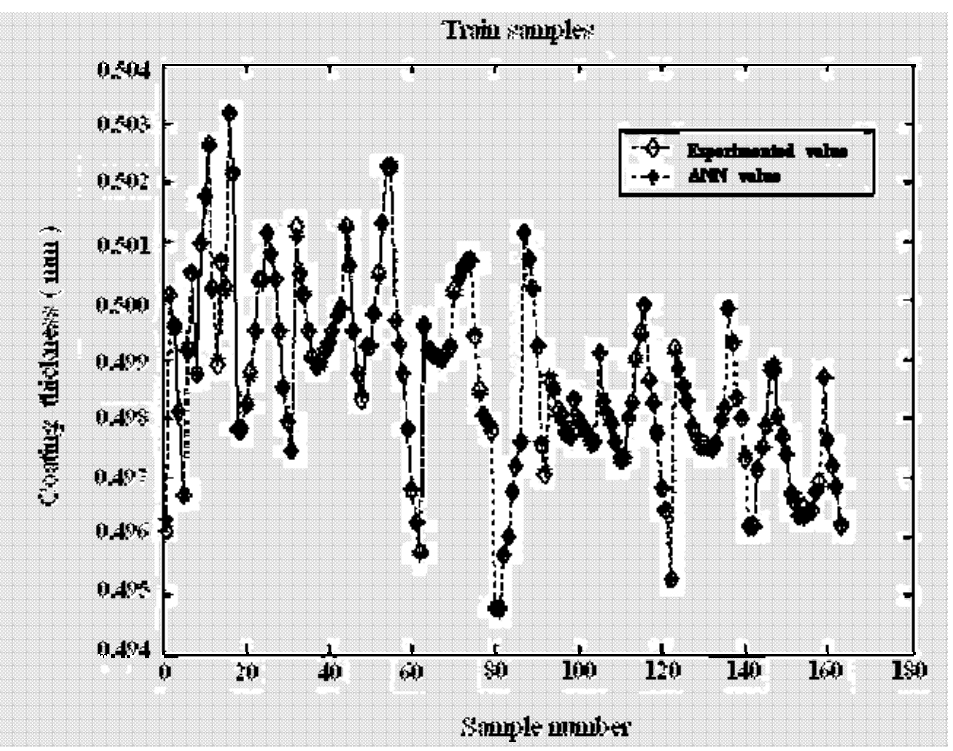

Figure 13. Train samples
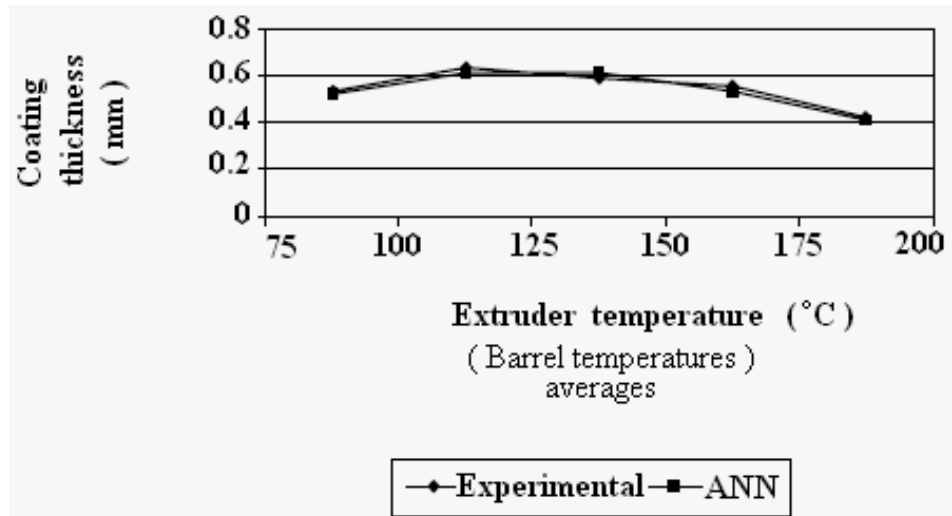

Figure 14. The comparison of coating thickness values with ANNs results

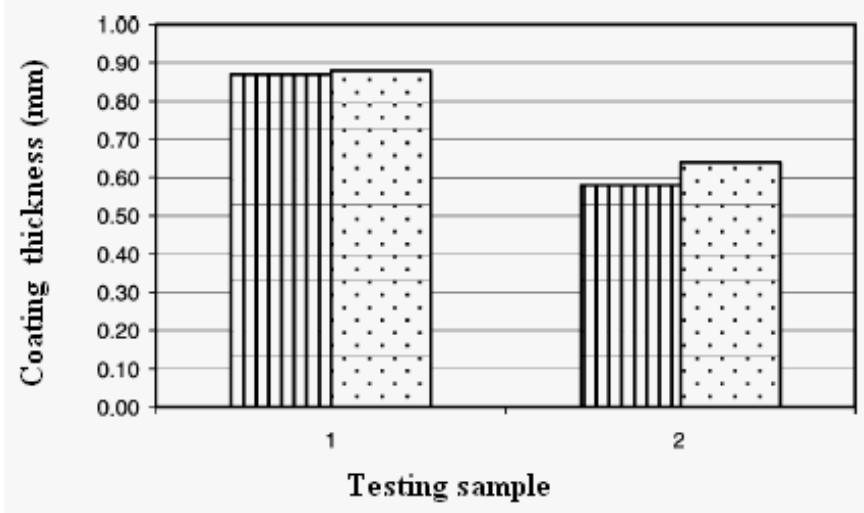

四 Experimental results $\square$ ANN results

Figure 15. The comparison of experimental coating thickness Values with ANNs results for testing samples 


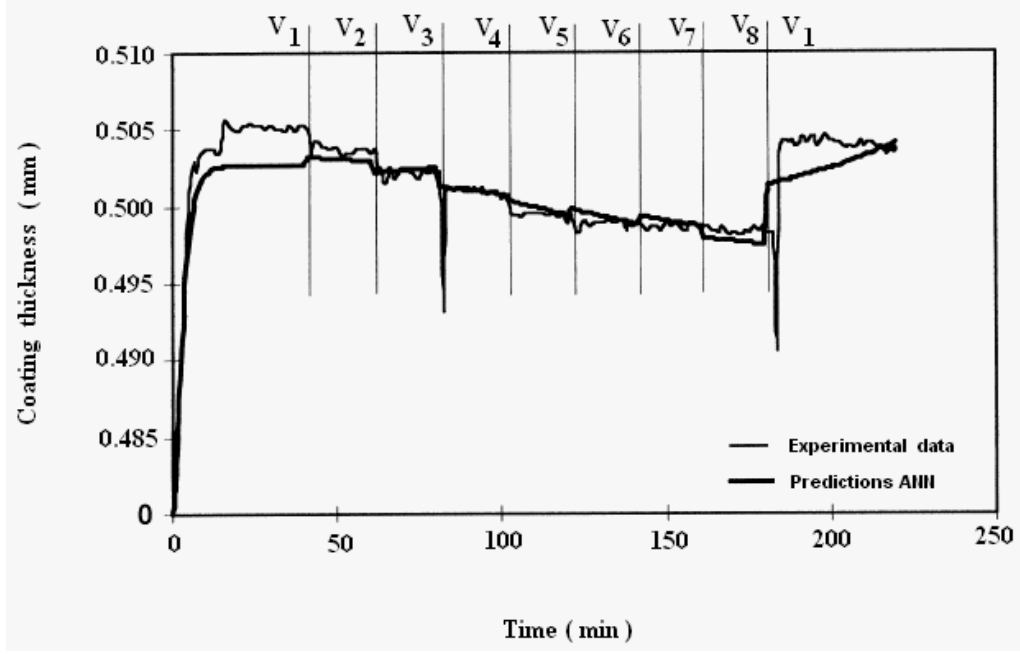

Figure 16. Experimental data and predictions of coating thickness by given full title ANN during extrusion

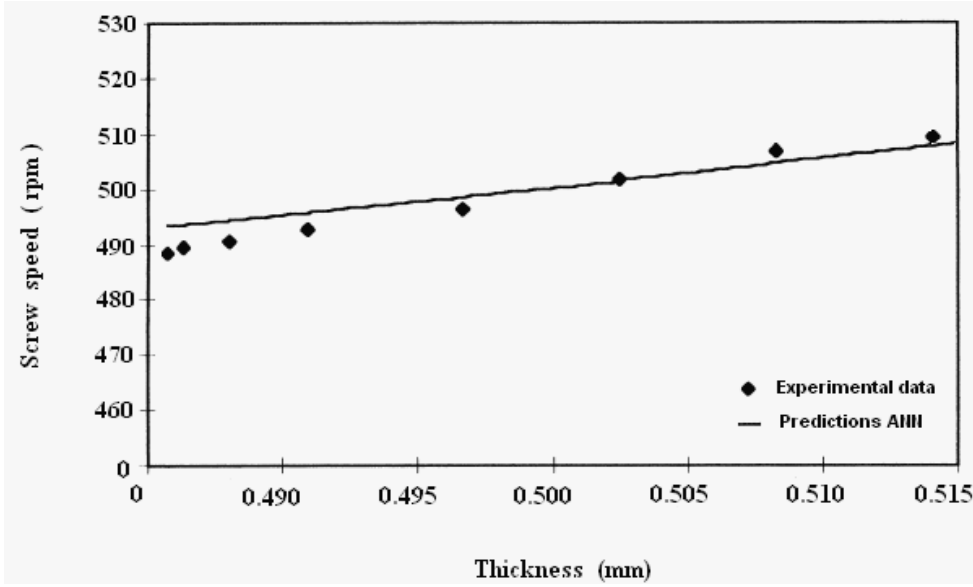

Figure 17. Experimental data and predictions of extruder screw speed with coating thickness by the $6-13-1$ ANN model

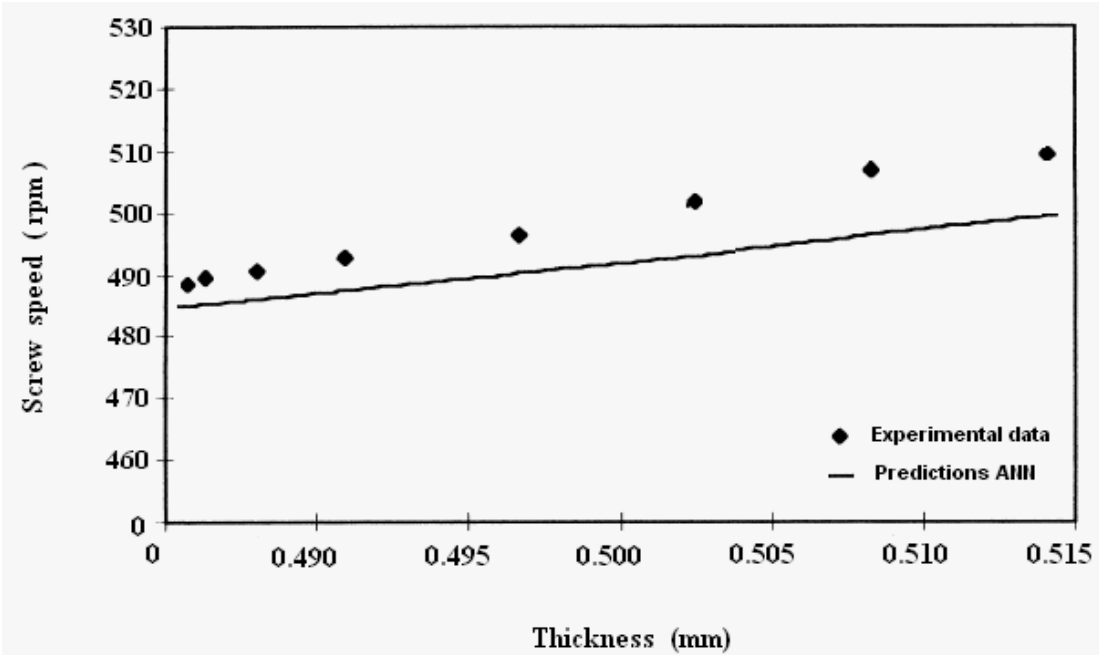

Figure 18. Experimental data and predictions of extruder screw speed with coating thickness by the $6-9-1$ ANN model 


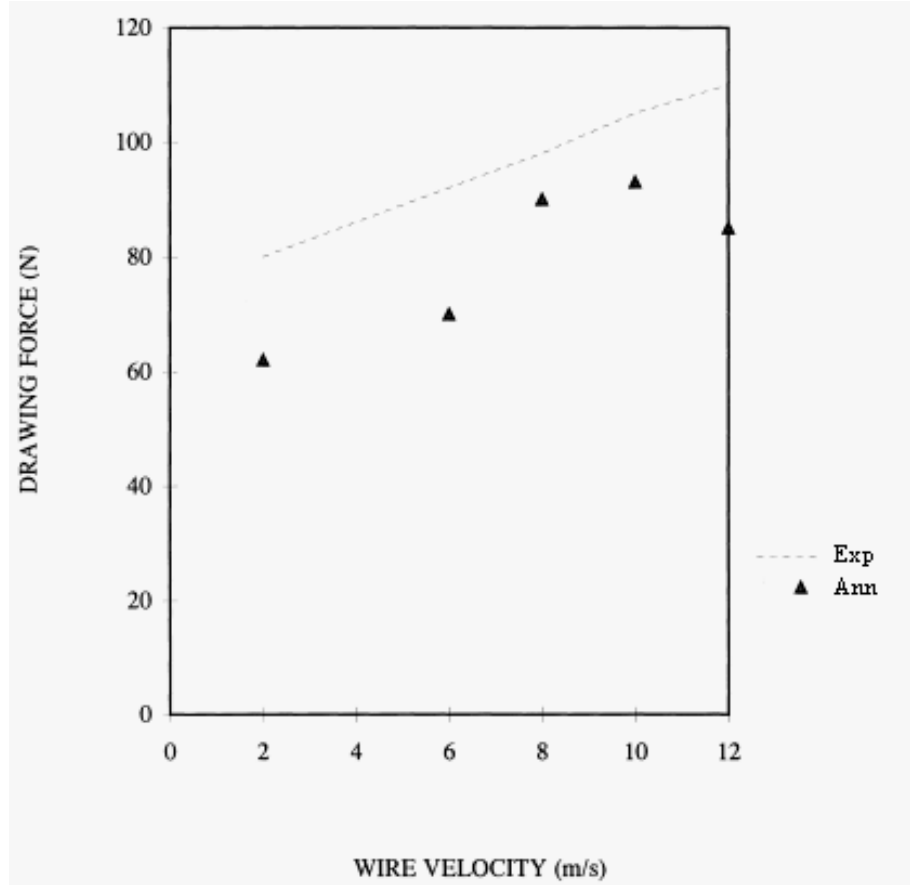

Figure 19. Drawing force for extruder temperature of $150 \circ \mathrm{C}$ ( Polimer - Ep $58 \mathrm{pvc}$ )

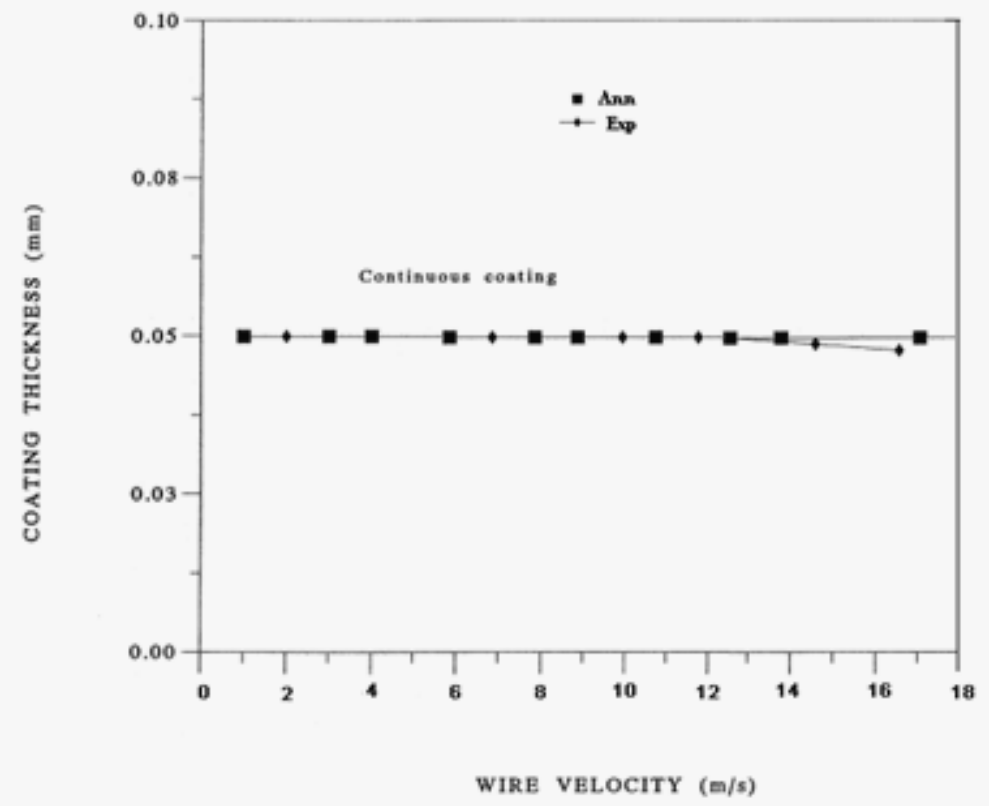

Figure 20. The relationship of wire velocity with coating thickness 